\title{
DISTRIBUIÇÃO DOS FONOAUDIÓLOGOS NA ATENÇÃO À SAÚDE NO ESTADO DE MINAS GERAIS ENTRE 2005 E 2010
}

\author{
Distribution of speech therapists in health care in Minas Gerais \\ state between 2005 and 2010
}

\author{
Camila Lúcia Ferreira (1), Fábio Rocha da Silva (2), Vanessa de Oliveira Martins-Reis ${ }^{(3)}$, \\ Amélia Augusta de Lima Friche ${ }^{(4)}$, Juliana Nunes Santos ${ }^{(5)}$
}

\begin{abstract}
RESUMO
Objetivo: analisar a tendência de distribuição dos fonoaudiólogos inseridos na atenção à saúde no estado de Minas Gerais no período de 2005 a 2010. Método: trata-se de um estudo retrospectivo, baseado na análise de banco de dados secundários de domínio público, os Cadernos de Informações de Saúde do Sistema de Informações em Saúde Brasileiro, o DATASUS. Foram analisados dados referentes aos 853 municípios do estado, destacando-se os indicadores de recursos relacionados ao número de profissionais de fonoaudiologia, o número de profissionais nas macrorregiões do estado, a população destes espaços, a vinculação dos fonoaudiólogos ao Sistema Único de Saúde (SUS) e as tendências de distribuição no período pesquisado. Resultados: no período de 2005 a 2010, houve significante crescimento do número de fonoaudiólogos. O número de municípios com fonoaudiólogos passou de $32,8 \%$ para $54,5 \%$. A partir de 2008 , ano marcado como o auge do crescimento do profissional vinculado ao SUS, observou-se aumento de cerca de $25 \%$. O número de profissionais por 10.000 habitantes passou de 0,61 em 2005 para 1,30 em 2010. Conclusão: observou-se aumento considerável na distribuição dos profissionais de fonoaudiologia inseridos na atenção à saúde no estado de Minas Gerais no período em questão, passando de 1.237 em 2005 a 2.555 em 2010. Em 2005, 278 municípios possuíam o profissional fonoaudiólogo na atenção à saúde. Cinco anos depois se observou aumento de 186 municípios com fonoaudiólogo, indicando maior disponibilidade deste profissional para a população atendida. Ainda que tais dados reforcem a maior acessibilidade e presença do fonoaudiólogo no estado, foi notória a discrepância regional no estado.
\end{abstract}

DESCRITORES: Fonoaudiologia; Sistema Único de Saúde; Programa Saúde da Família; Saúde Pública; Políticas Públicas

(1) Acadêmica do curso de Fonoaudiologia da Universidade Federal de Minas Gerais.

(2) Acadêmico do curso de Fonoaudiologia da Universidade Federal de Minas Gerais;

(3) Fonoaudióloga; Professora adjunto do curso de Fonoaudiologia da Universidade Federal de Minas Gerais - UFMG, Belo Horizonte, MG, Brasil; Doutora em Saúde Pública pela UFMG.

(4) Fonoaudióloga; Professora adjunto do curso de Fonoaudiologia da Universidade Federal de Minas Gerais UFMG, Belo Horizonte, MG, Brasil; Doutora em Linguística pela Universidade de São Paulo.

(5) Fonoaudióloga; Professora adjunto do curso de Fonoaudiologia da Universidade Federal de Minas Gerais - UFMG, Belo Horizonte, MG, Brasil; Doutora em Ciências da saúde pela UFMG.

Trabalho realizado no Departamento de Fonoaudiologia da Faculdade de Medicina da Universidade Federal de Minas Gerais - UFMG - Minas Gerais (MG), Brasil.

Conflito de interesses: inexistente

\section{INTRODUÇÃO}

A inserção da Fonoaudiologia no vasto campo das ciências da saúde é um evento relativamente recente, especialmente se comparada à história de outras tantas ciências consolidadas ${ }^{1,2}$. Contudo, o cenário delineado após pouco mais de três décadas de atuação da Fonoaudiologia, mostra grupos de profissionais cada vez mais atuantes e integrados a equipes multiprofissionais e aos diversos níveis de atendimento à saúde das populações, corroborando com as transformações das diretrizes da saúde coletiva nos últimos 25 anos, que influenciam também mudanças na concepção de saúde e no seu modelo de organização ${ }^{3}$. 
O panorama profissional que se desenrola nos dias atuais pode ser percebido como fruto da mobilização de uma classe em favor de seu reconhecimento, mas também do surgimento de avanços científicos que respaldam a prática clínica fonoaudiológica e justificam a presença destes profissionais nos ambientes de promoção de saúde. Não obstante, há de se considerar as influências das novas perspectivas e propostas relacionadas às políticas de saúde, condição fundamental para se discutir o destaque crescente da Fonoaudiologia e sua melhor representatividade em relação ao SUS, na medida em que pode ser reconhecida uma relação significante entre esse crescimento e a criação de políticas de saúde, como a Política Nacional de Atenção à Saúde Auditiva ${ }^{4}$ e o Núcleo de Apoio a Saúde da Família (NASF) ${ }^{5}$.

De acordo com os princípios norteadores do SUS, a Fonoaudiologia deve atuar nos três níveis de atenção: primário, secundário e terciário. Os enfoques do nível primário visam à prevenção, à reabilitação e promoção associada à saúde dos indivíduos. Nos níveis secundário e terciário, pode ser observada, por vezes, uma abordagem com foco mais curativo, perspectiva esta que também vem sendo modificada nos últimos anos, a partir do surgimento de novas propostas em saúde ${ }^{6}$

No que se refere ao nível primário, a iniciativa de criação do NASF, por meio da Portaria nำ154, de 24 de janeiro de 2008, teve por objetivo ampliar as condições e a qualidade do atendimento nos segmentos da atenção básica, favorecendo a aproximação e diálogo de diversas especialidades responsáveis por diagnosticar problemas ambientais passíveis de intervenção dos diversos setores inseridos neste programa. As propostas do NASF contribuem para que a atuação fonoaudiológica seja conhecida pelos profissionais das equipes de Saúde da Família e também para que a população seja beneficiada com as ações de promoção, prevenção e reabiltação que estes profissionais desenvolvem nas comunidades ${ }^{7}$

Considerando-se a magnitude das ações que o profissional pode desempenhar, tem-se como condição básica para o desenvolvimento de uma prática efetiva, a formação de profissionais críticos que reconheçam as demandas da população, bem como a realidade da saúde pública, que sejam conscientes de que a promoção de saúde é algo maior do que a simples prevenção de doenças, e que sejam capazes de atender e encaminhar adequadamente os diversos pacientes que os procuram ${ }^{8,9}$

No estado de Minas Gerais, a inserção do profissional de Fonoaudiologia ocorreu, inicialmente, com o propósito de promover uma atuação voltada para a execução de práticas curativas. Os profissionais que, no começo da década de 1970, se propuseram a trabalhar nos serviços em saúde de Minas Gerais, estavam vinculados a instituições e clínicas conveniadas. Estes profissionais deram início à formação de grupos organizados responsáveis pela difusão de sua atuação e, posteriormente, colaboraram para a regulamentação da fonoaudiologia, no ano de 1981. Eles contribuíram não apenas para difusão da fonoaudiologia enquanto ciência, mas também para a estruturação de um novo sistema de atendimento à saúde ${ }^{10}$.

A tentativa de delinear o perfil da distribuição dos profissionais e de caracterizá-los permite alimentar a discussão sobre o impacto das políticas propostas e analisar, sob uma nova perspectiva, uma série de fatores relacionados às tendências que permeiam estes processos. Estudos como este e as reflexões que geram contribuem para a criação e implementação de propostas mais eficazes que contemplem demandas populacionais e cumpram com os princípios delineados na criação do SUS ${ }^{8}$.

O objetivo deste estudo é analisar a tendência de distribuição dos profissionais de Fonoaudiologia na atenção à saúde no estado de Minas Gerais no período de 2005 a 2010.

\section{MÉTODO}

Trata-se de um estudo retrospectivo, baseado na análise do banco de dados secundários de domínio público. Foi realizado no período de março a dezembro de 2011. Os dados analisados nesta pesquisa foram obtidos por meio da consulta aos Cadernos de Informações de Saúde do Sistema de Informações em Saúde Brasileiro, o DATASUS, em sua versão mais atualizada. Foram analisados os dados dos últimos cinco anos (2005 a 2010).

$\mathrm{Na}$ pesquisa, foram analisados dados referentes aos 853 municípios do estado de Minas Gerais, destacando-se os indicadores de recurso relacionados a: número de profissionais de Fonoaudiologia na atenção à saúde, número de profissionais nas macro e microregiões do estado, população destes espaços, vinculação dos fonoaudiólogos ao Sistema Único de Saúde (SUS) e tendências de distribuição deste profissional no período de 2005 a 2010.

Após a coleta, foram analisadas as seguintes variáveis: presença do profissional de Fonoaudiologia no município, número de fonoaudiólogos atuantes e sua relação com a população dos municípios, a distribuição espacial dos profissionais por macrorregiões do estado, bem como a inserção do fonoaudiólogo no SUS.

Essa pesquisa foi aprovada pelo Comitê de Ética em Pesquisa da Universidade Federal de Minas Gerais, sob protocolo 0483-10. 
Os dados obtidos foram armazenados e analisados em banco de dados eletrônico. Para fins de análise descritiva, procedeu-se à distribuição de frequência das variáveis categóricas envolvidas na distribuição das variáveis em estudo e à análise das medidas de tendência central e de dispersão das variáveis contínuas. $\mathrm{Na}$ análise estatística inferencial foi empregados o teste Qui-quadrado e o Teste T, de Student.

\section{RESULTADOS}

Foram analisados os dados dos 853 municípios mineiros no período de 2005 a 2010. O número de municípios mineiros com a presença do fonoaudiólogo na rede de saúde (pública ou privada) pode ser visualizado na Tabela 1.

A Tabela 2 mostra a evolução da inserção do profissional fonoaudiólogo no Sistema de Saúde mineiro no período considerado.

A Figura 1 revela a relação que se estabelece entre o número de profissionais fonoaudiólogos e a dimensão populacional das macrorregionais do estado de Minas Gerais.

A Figura 2 ilustra a tendência de distribuição do fonoaudiólogo no estado no período de 2005 a 2010.

A Tabela 3 mostra a disponibilidade de profissionais nos municípios mineiros de acordo com suas dimensões populacionais.

Tabela 1 - Análise cronológica da presença do profissional fonoaudiólogo nos municípios do estado de Minas Gerais no período de 2005 a 2010

\begin{tabular}{ccccccc}
\hline \multirow{2}{*}{ Ano } & \multicolumn{2}{c}{$\begin{array}{c}\text { Municípios com } \\
\text { Fonoaudiólogo }\end{array}$} & \multicolumn{2}{c}{$\begin{array}{c}\text { Municípios sem } \\
\text { Fonoaudiólogo }\end{array}$} & \multicolumn{2}{c}{$\begin{array}{c}\text { Municípios com } \\
\text { Fonoaudiólogo na rede } \\
\text { sus }\end{array}$} \\
\cline { 2 - 7 } & $\mathbf{n}$ & $\%$ & $\mathbf{n}$ & $\%$ & $\mathbf{n}$ & $\%$ \\
\hline 2005 & 280 & $32,8 \%$ & 573 & $67,2 \%$ & 271 & $31,7 \%$ \\
2006 & 320 & $37,5 \%$ & 533 & $62,4 \%$ & 315 & $36,9 \%$ \\
2007 & 350 & $41,0 \%$ & 503 & $58,9 \%$ & 341 & $39,9 \%$ \\
2008 & 393 & $46,0 \%$ & 460 & $53,9 \%$ & 379 & $44,4 \%$ \\
2009 & 450 & $52,7 \%$ & 403 & $47,2 \%$ & 433 & $50,7 \%$ \\
2010 & 465 & $54,5 \%$ & 388 & $45,4 \%$ & 449 & $52,6 \%$ \\
\hline
\end{tabular}

Fonte: Cadernos de Informações de saúde, versão de maio de 2010. DATA SUS, Ministério da Saúde.

Tabela 2 - Número de profissionais fonoaudiólogos vinculados ao Sistema Único de Saúde no estado de Minas Gerais no período de 2005 a 2010, segundo as macroregionais de saúde

\begin{tabular}{ccccccc}
\hline Macrorregião do estado & $\mathbf{2 0 0 5}$ & $\mathbf{2 0 0 6}$ & $\mathbf{2 0 0 7}$ & $\mathbf{2 0 0 8}$ & $\mathbf{2 0 0 9}$ & $\mathbf{2 0 1 0}$ \\
\hline Sul & 232 & 246 & 240 & 251 & 278 & 291 \\
Centro-Sul & 57 & 76 & 98 & 121 & 110 & 107 \\
Centro & 213 & 241 & 314 & 456 & 521 & 542 \\
Jequitinhonha & 6 & 10 & 14 & 13 & 22 & 22 \\
Oeste & 47 & 56 & 63 & 73 & 86 & 91 \\
Leste & 34 & 43 & 38 & 51 & 70 & 73 \\
Sudeste & 88 & 105 & 106 & 143 & 162 & 171 \\
Norte de Minas & 52 & 60 & 71 & 85 & 104 & 100 \\
Noroeste & 37 & 40 & 39 & 42 & 44 & 44 \\
Leste do Sul & 19 & 25 & 34 & 40 & 52 & 54 \\
Nordeste & 18 & 20 & 19 & 29 & 39 & 40 \\
Triângulo do Sul & 64 & 70 & 67 & 82 & 80 & 82 \\
Triângulo do Norte & 53 & 62 & 64 & 69 & 68 & 75 \\
\hline MINAS GERAIS & 920 & 1054 & 1167 & 1455 & 1636 & 1692 \\
\hline
\end{tabular}

Fonte: Cadernos de Informações de saúde, versão de maio de 2010. DATA SUS, Ministério da Saúde. 


\begin{tabular}{|c|c|c|c|c|c|c|c|c|c|c|c|c|}
\hline \multirow{3}{*}{ Macrorregional } & \multicolumn{12}{|c|}{ Número de profissionais fonoaudiólogos $(\mathrm{N})$ e média por 10.000 habitantes $(\mathrm{X})$} \\
\hline & \multicolumn{2}{|c|}{2005} & \multicolumn{2}{|c|}{2006} & \multicolumn{2}{|c|}{2007} & \multicolumn{2}{|c|}{2008} & \multicolumn{2}{|c|}{2009} & \multicolumn{2}{|c|}{2010} \\
\hline & $\mathbf{N}$ & $\mathbf{X}$ & $\mathbf{N}$ & $\mathbf{X}$ & $\mathbf{N}$ & $\mathbf{X}$ & $\mathbf{N}$ & $\mathbf{X}$ & $\mathbf{N}$ & $\mathbf{X}$ & $\mathbf{N}$ & $\mathbf{X}$ \\
\hline Sul & 271 & 1,04 & 304 & 1,15 & 338 & 1,26 & 377 & 1,44 & 414 & 1,57 & 431 & 1,65 \\
\hline Centro-Sul & 74 & 1,04 & 101 & 1,41 & 121 & 1,68 & 158 & 2,17 & 156 & 2,12 & 153 & 2,11 \\
\hline Centro & 370 & 0,60 & 468 & 0,75 & 578 & 0,91 & 756 & 1,20 & 869 & 1,36 & 915 & 1,49 \\
\hline Jequitinhonha & 6 & 0,21 & 10 & 0,35 & 14 & 0,49 & 17 & 0,57 & 25 & 0,84 & 25 & 0,87 \\
\hline Oeste & 59 & 0,52 & 79 & 0,68 & 93 & 0,79 & 77 & 0,64 & 122 & 1,01 & 130 & 1,09 \\
\hline Leste & 54 & 0,38 & 70 & 0,49 & 75 & 0,52 & 106 & 0,72 & 129 & 0,88 & 137 & 0,94 \\
\hline Sudeste & $\mathrm{c}$ & 0,59 & 114 & 0,73 & 149 & 0,94 & 195 & 1,23 & 222 & 1,39 & 234 & 1,49 \\
\hline Norte de Minas & 52 & 0,33 & 64 & 0,41 & 98 & 0,62 & 134 & 0,83 & 158 & 0,98 & 160 & 1,01 \\
\hline Noroeste & 40 & 0,62 & 46 & 0,71 & 43 & 0,65 & 52 & 0,79 & 55 & 0,83 & 56 & 0,85 \\
\hline Leste do Sul & 21 & 0,31 & 26 & 0,39 & 37 & 0,55 & 48 & 0,71 & 61 & 0,90 & 63 & 0,94 \\
\hline Nordeste & 18 & 0,20 & 20 & 0,22 & 19 & 0,21 & 29 & 0,31 & 42 & 0,45 & 44 & 0,48 \\
\hline Triângulo do Sul & 71 & 1,11 & 78 & 1,20 & 80 & 1,22 & 98 & 1,42 & 92 & 1,31 & 96 & 1,37 \\
\hline $\begin{array}{c}\begin{array}{c}\text { Triângulo do } \\
\text { Norte }\end{array} \\
\end{array}$ & 60 & 0,52 & 85 & 0,73 & 88 & 0,74 & 97 & 0,80 & 102 & 0,83 & 111 & 0,94 \\
\hline Minas Gerais & 1187 & 0,61 & 1465 & 0,75 & 1733 & 0,87 & 2174 & 1,09 & 2447 & 1,22 & 2555 & 1,30 \\
\hline
\end{tabular}

Fonte: Cadernos de Informações de saúde, versão de maio de 2010. DATA SUS, Ministério da Saúde.

Figura 1 - Relação entre o número de profissionais fonoaudiólogos e a dimensão populacional das macrorregionais do estado de Minas Gerais no período de 2005 a 2010
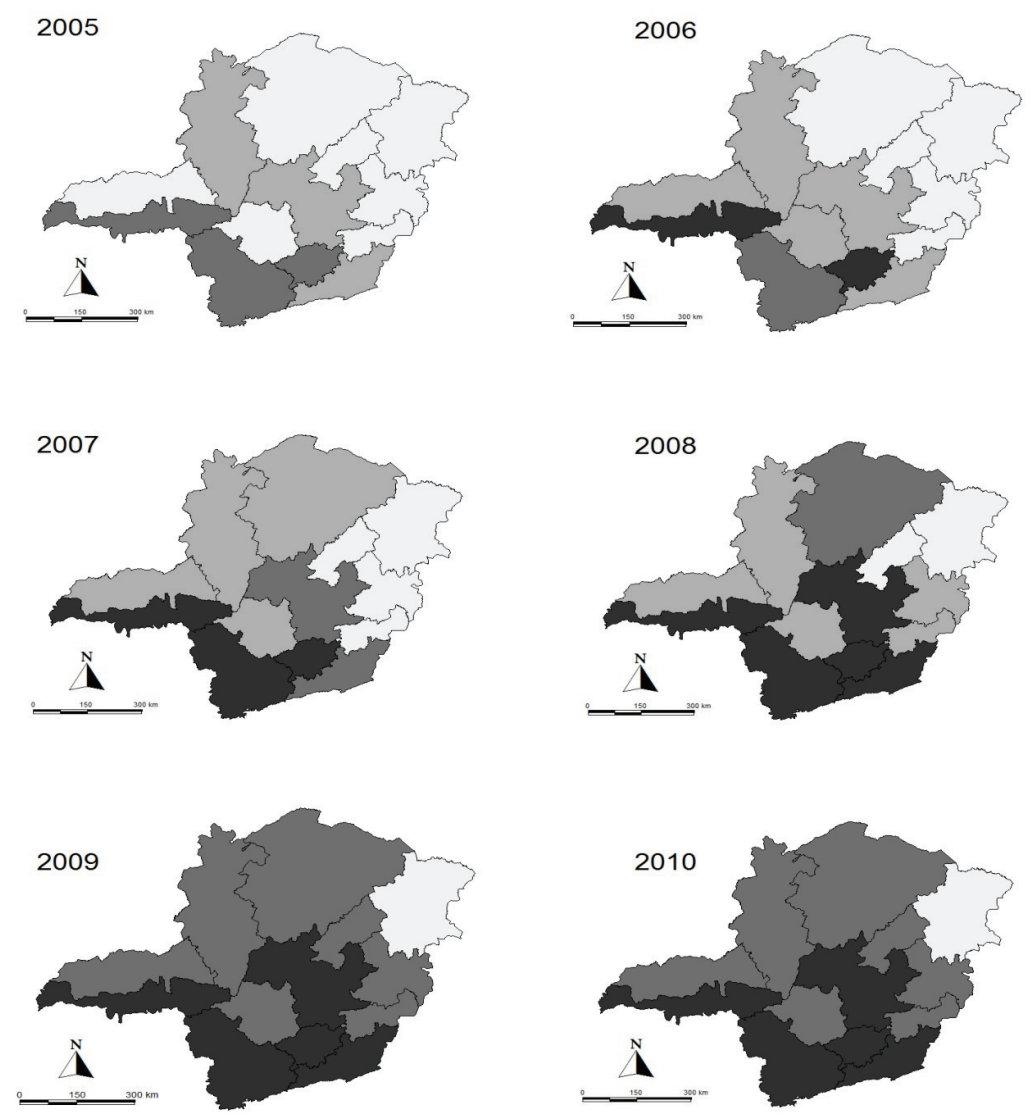

Fonoaudiógos/10.000 hab

1,19 to $2,17 \square 0,83$ to $1,19 \square 0,58$ to $0,83 \square 0,2$ to 0,58

Fonte: Cadernos de Informações de saúde, versão de maio de 2010. DATA SUS, Ministério da Saúde.

Figura 2 - Tendência de distribuição do profissional fonoaudiólogo vinculado ao SUS no estado no período de 2005 a 2010 
Tabela 3 - Distribuição do número de fonoaudiólogos nos municípios, segundo o tamanho populacional no período de 2005 a 2010

\begin{tabular}{|c|c|c|c|c|c|c|c|c|c|c|c|}
\hline \multirow[b]{2}{*}{ Ano } & & \multicolumn{10}{|c|}{ Cidades mineiras por número de habitantes } \\
\hline & & $\begin{array}{c}\text { Até } 5.000 \\
\text { Hab } \\
(n=225)\end{array}$ & $\%$ & $\begin{array}{c}\text { Entre } 5 \text { e } \\
10.000 \mathrm{hab} \\
(\mathrm{n}=264)\end{array}$ & $\%$ & $\begin{array}{c}\text { Entre } 10 \mathrm{e} \\
50.000 \mathrm{hab} \\
(\mathrm{n}=299)\end{array}$ & $\%$ & $\begin{array}{c}\text { Entre } 50 \text { e } \\
100.000 \\
\text { hab }(n=38)\end{array}$ & $\%$ & $\begin{array}{c}\text { Acima de } \\
100.000 \\
(n=27) \\
\end{array}$ & $\%$ \\
\hline \multirow[b]{2}{*}{2005} & fonoaudiólogo & 29 & $12,8 \%$ & 57 & $21,5 \%$ & 130 & $43,4 \%$ & 37 & $97,3 \%$ & 27 & $100 \%$ \\
\hline & $\begin{array}{l}\text { Fonoaudiólogo } \\
\text { na rede sus }\end{array}$ & 28 & $12,4 \%$ & 55 & $20,8 \%$ & 126 & $42,1 \%$ & 36 & $94,7 \%$ & 26 & $96,2 \%$ \\
\hline \multirow[b]{2}{*}{2006} & fonoaudiólogo & 35 & $15,5 \%$ & 67 & $25,3 \%$ & 154 & $51,5 \%$ & 37 & $97,3 \%$ & 27 & $100 \%$ \\
\hline & $\begin{array}{l}\text { Fonoaudiólogo } \\
\text { na rede sus }\end{array}$ & 35 & $15,5 \%$ & 66 & $25 \%$ & 151 & $50,5 \%$ & 36 & $94,7 \%$ & 27 & $100 \%$ \\
\hline \multirow[b]{2}{*}{2007} & fonoaudiólogo & 42 & $18,6 \%$ & 74 & $28 \%$ & 170 & $56,8 \%$ & 37 & $97,3 \%$ & 27 & $100 \%$ \\
\hline & $\begin{array}{l}\text { Fonoaudiólogo } \\
\text { na rede sus }\end{array}$ & 42 & $18,6 \%$ & 71 & $26,8 \%$ & 164 & $54,8 \%$ & 37 & $97,3 \%$ & 27 & $100 \%$ \\
\hline \multirow[b]{2}{*}{2008} & fonoaudiólogo & 53 & $23,5 \%$ & 80 & $30,3 \%$ & 195 & $65,2 \%$ & 38 & $100 \%$ & 27 & $100 \%$ \\
\hline & $\begin{array}{l}\text { Fonoaudiólogo } \\
\text { na rede sus }\end{array}$ & 51 & $22,6 \%$ & 76 & $28,7 \%$ & 187 & $62,5 \%$ & 38 & $100 \%$ & 27 & $100 \%$ \\
\hline \multirow[b]{2}{*}{2009} & fonoaudiólogo & 66 & $29,3 \%$ & 98 & $37,1 \%$ & 221 & $73,9 \%$ & 38 & $100 \%$ & 27 & $100 \%$ \\
\hline & $\begin{array}{l}\text { Fonoaudiólogo } \\
\text { na rede sus }\end{array}$ & 64 & $28,4 \%$ & 95 & $35,9 \%$ & 209 & $69,8 \%$ & 38 & $100 \%$ & 27 & $100 \%$ \\
\hline \multirow[b]{2}{*}{2010} & fonoaudiólogo & 74 & $32,8 \%$ & 104 & $39,3 \%$ & 222 & $74,2 \%$ & 38 & $100 \%$ & 27 & $100 \%$ \\
\hline & $\begin{array}{l}\text { Fonoaudiólogo } \\
\text { na rede sus }\end{array}$ & 72 & $32 \%$ & 101 & $38,2 \%$ & 211 & $70,5 \%$ & 38 & $100 \%$ & 27 & $100 \%$ \\
\hline
\end{tabular}

Fonte: Cadernos de Informações de saúde, versão de maio de 2010. DATA SUS, Ministério da Saúde.

\section{DISCUSSÃO}

Este trabalho analisou a evolução do número de profissionais de fonoaudiologia inseridos na atenção à saúde no estado de Minas Gerais com base na consulta aos Cadernos de Informações de Saúde do Sistema de Informações em Saúde Brasileiro, o DATASUS. A utilização de informações disponíveis em bancos de dados secundários tem sido frequente na medida em que tem contribuído para estruturação de programas de grande alcance e para mensurar os impactos de propostas já implementadas na esfera da saúde pública. A literatura revela que pesquisas envolvendo estas fontes de informações são realizadas em diversas áreas ${ }^{11}$ e que muitas vezes, orientam a aplicação de recursos, direcionando-os de acordo com a situação de saúde das populações atendidas. As bases de dados secundários permitem traçar 0 perfil de mudanças empreendidas ao longo de anos e mensurar os impactos de fatores relacionados aos objetos de estudo de maneira segura e efetiva. A Fonoaudiologia, ciência relativamente nova, também tem utilizado, em favor do desenvolvimento de pesquisas, fontes de dados públicos, sendo notória sua contribuição para a estruturação de políticas importantes, como o Programa de Saúde Auditiva, e para o monitoramento do modelo de organização vigente e dos resultados obtidos ${ }^{12}$.
Os dados encontrados durante a realização deste estudo demonstram que no período considerado, entre 2005 e 2010, houve um crescimento significante do número de profissionais de Fonoaudiologia no estado de Minas Gerais. Os achados são contundentes ao revelarem a evolução do número de municípios que contam com este profissional em seus serviços de saúde, passando de $32,8 \%$ em 2005 a 54,5\% em 2010 (Tabela1). Devem-se destacar, no entanto, os dados referentes aos anos de 2008 e 2009, nos quais se observa uma mudança substancial no número de municípios que passaram a possuir serviços de Fonoaudiologia, o que pode estar relacionado ao reconhecimento da importância da profissão nas políticas públicas de saúde ${ }^{7,13,14}$.

O início deste processo de expansão tem raízes distantes já no ano de 2002, quando propostas destinadas à inclusão da Fonoaudiologia como componente da equipe responsável pelo atendimento na Atenção Básica foram formuladas pelo Conselho Regional de Fonoaudiologia da $2^{2}{ }^{a}$ Região ${ }^{7}$ Desde então, os gestores em saúde têm implementado políticas de amplo alcance, garantindo o acesso da população a serviços antes disponíveis apenas nos grandes centros urbanos e pólos regionais. No que se refere a estas políticas desenvolvidas, verifica-se cada vez mais a presença de profissionais como os fonoaudiólogos, sendo possível citar como exemplo importante a Política Nacional de Atenção à Saúde Auditiva ${ }^{4}$. 
É possível constatar a ocorrência da evolução da inserção do fonoaudiólogo no Sistema Único de Saúde (SUS) a partir de 2008, ano marcado como o auge do crescimento do profissional vinculado ao SUS, sendo este crescimento estimado em 25\% (Figura 1). O padrão de crescimento observado neste período pode ser considerado um reflexo das novas políticas de saúde instituídas pelo Governo Federal como os Núcleos de Apoio à Saúde da Família (NASF) ${ }^{5,12}$, implementados por meio da Portaria 154, de 2008, cujo objetivo era promover a ampliação das ações desenvolvidas na Atenção Básica e o alcance destas no âmbito das comunidades atendidas. O movimento empreendido nesta época por meio deste instrumento político ilustra a mudança no modo de organizar e de gerenciar o atendimento à saúde, bem como de colocar efetivamente em prática os princípios doutrinários do Sistema Único de Saúde ${ }^{3}$

Estudo anterior ${ }^{15}$, baseado no número de fonoaudiólogos por 10.000 habitantes ${ }^{16}$, apontou que seriam necessários 2.600 fonoaudiólogos atuantes na rede pública de saúde no estado de Minas Gerais, sendo 2.000 na rede básica, 400 na média complexidade e 200 na alta complexidade. Dados revelam que a partir de 2017 o estado terá número adequado de fonoaudiólogos/10.000hab. Para um cálculo mais preciso, é necessário levar em conta a jornada de trabalho de cada um desses profissionais. Com a possível aprovação da Lei Federal 2.192/03, a jornada de trabalho do fonoaudiólogo passará a ser de $30 \mathrm{~h}$ semanais ${ }^{17}$. Estimativas realizadas consideraram que o profissional atua $40 \mathrm{~h}$ no SUS. Além disso, deve-se levar em conta a região de alocação de cada um desses profissionais, uma vez que os resultados mostraram uma disparidade entre as macrorregiões do estado.

As informações disponibilizadas no Cadastro Nacional dos Estabelecimentos de Saúde do Brasil (CNES) corroboram com os números obtidos e a associação estabelecida entre a política do NASF, vigente a partir de 2008, e a ampliação do número de municípios que contam com fonoaudiólogos. Os dados disponíveis revelam a dimensão em que ocorreu o aumento destes núcleos por todo o estado a partir dos meses seguintes a sua implantação e, consequentemente, do número de profissionais disponíveis.

A média de fonoaudiólogos por 10.000 habitantes passou de 0,61 em 2005 para 1,30 em 2010 (Quadro 1), indicando maior disponibilidade deste profissional para a população mineira. A análise realizada sobre a disponibilidade de outros profissionais da área da saúde revela que a média de profissionais odontólogos por 10.000 habitantes passou de 13,4 em 2005 para 13,9 em 2008. Já os profissionais de nutrição, profissão também contemplada nos programas de saúde coletiva, cresceu de 0,7 profissionais por 10.000 habitantes em 2005 para 1,4 no ano de $2008^{18}$. Esta informação reafirma a hipótese de que programas governamentais que buscam colocar em prática a assistência universal e integral à saúde beneficiam o profissional de reabilitação e permite que estes adequem suas práticas às novas tendências e demandas da atenção básica à saúde.

Observa-se, no entanto, que não apenas as mudanças no âmbito das políticas de saúde podem ser associadas a este aumento do número de profissionais. É possível destacar também a demanda crescente dos usuários que se apresentam nas unidades de saúde ${ }^{19}$. Com o passar dos anos, verificou-se que a Fonoaudiologia se firmou como ciência, com base em pesquisas e estudos que possibilitaram avanços expressivos na prática profissional e permitiram que esta se tornasse conhecida a outros profissionais da saúde e àqueles que buscam os serviços de atenção à saúde ${ }^{1}$.

Por sua história recente a Fonoaudiologia, que até então não fazia parte das ações de Atenção Básica à Saúde, viu surgir a necessidade de se adaptar a uma nova prática, voltada não ao atendimento clínico privado, mas, sim ao contexto histórico-cultural do indivíduo usuário do sistema público de saúde ${ }^{20,21}$ Observa-se, a partir deste momento um forte movimento de mudança, que se inicia na academia, na medida em que se verifica uma mobilização pela reestruturação dos currículos e das propostas de ensino voltadas, agora para a formação de profissionais aptos a lidar com esta nova demanda ${ }^{21}$ e para a mudança nas perspectivas dos profissionais atuantes. Considerar a mudança empreendida neste momento é fator importante para analisar os achados deste estudo, já que esta não apenas repercute na melhor preparação dos futuros profissionais, como também desperta seu interesse pela atuação nesta área tão ampla e promissora ${ }^{22}$. Seguindo as trilhas desta mudança, há que se levar em conta também os impactos gerados na própria estrutura e funcionamento do sistema, pois é clara a relação entre a efetividade das propostas dos órgãos gestores e a preparação dos profissionais ${ }^{23}$.

Ainda que os dados obtidos neste estudo reforcem a maior acessibilidade e presença do profissional de Fonoaudiologia no estado de Minas Gerais, os dados revelam que existe uma discrepância considerável em sua distribuição (Tabela 2) e o número de fonoaudiólogos atuantes na atenção à saúde ainda não é suficiente para a demanda populacional. A análise do período de 2005 a 2010 mostra que a macrorregião Centro é a que em 2005 
tinha o maior número de fonoaudiólogos, sendo notável o crescimento deste número em todos os anos conseguintes. Nos dados apresentados no mapa, verifica-se que as macrorregiões Centro, Sul e Sudeste do estado também demonstram aumento considerável do número de profissionais fonoaudiólogos. Porém, estes índices são discretos quando comparados à região Centro-sul do estado. Em estudo recente, observou-se que no ano de 2009 as macrorregiões Centro-Sul e Sul apresentaram a maior média de fonoaudiólogos por 10 mil habitantes ${ }^{15}$. As diferenças observadas, ainda que revelem aspectos relacionados aos serviços de saúde, refletem também as diferenças expressivas quanto às características socioeconômicas e demográficas das regiões, sendo observado que as regiões Centro, Sul e Sudeste são detentoras dos melhores índices no que se refere a estas características ${ }^{24}$ A disparidade que se observa no estado também é vivenciada pelos serviços de Atenção à Saúde em outras regiões do mundo ${ }^{25}$, o que reforça a necessidade de se formular políticas que atuem na redução das barreiras quanto ao acesso das populações ao atendimento clínico amplo.

A análise da evolução histórica destas macrorregiões indica que em períodos anteriores ao contemplado nesta pesquisa esta disparidade se fazia presente, destacando-se as regiões Centro e Sul como aquelas que possuem a maior gama de serviços e profissionais. Estas regiões apresentam os melhores índices de alfabetização e de renda per capta do estado, o que leva a crer que quanto maior o nível de desenvolvimento de determinada região maiores suas possibilidades de implementação de políticas públicas de Atenção Básica à Saúde, tornando, assim, maiores as possibilidades de inserção de novos profissionais, especialmente os fonoaudiólogos no SUS. O fonoaudiólogo generalista encontra no SUS uma área de atuação potencial. Tendo esse conhecimento em mente, é necessário que os profissionais da classe não somente atuem de maneira clínica, mas também desenvolvam novos estudos que comprovem a importância da atuação fonoaudiológica na Atenção Básica à Saúde. Acredita-se que o momento é propício para a inserção do fonoaudiólogo no SUS na medida em que vários estudos demonstram a elevada ocorrência de distúrbios de comunicação em usuários do sistema público de saúde nacional e de outros países ${ }^{19,26}$ É necessário que sejam tomadas providências para suprir esta demanda e adequar o serviço de Fonoaudiologia às políticas de saúde vigentes ${ }^{6}$.
Ainda que os dados obtidos tenham permitido delinear as tendências de evolução do número de profissionais de Fonoaudiologia no estado, o panorama apresentado deve ser visto com atenção. Acredita-se que existe possível subnotificação no que se refere à presença destes profissionais no território. Estudos anteriores ${ }^{15}$ revelam a existência de desacordo entre as informações disponíveis nos Conselhos Regionais e no sistema consultado quanto ao número de profissionais que atuam em Minas Gerais. Considera-se, ainda, que a impossibilidade de obter informações sobre indicadores específicos da área pode representar um empecilho ao estabelecimento do panorama atual no que se refere à disponibilidade do profissional nos serviços de saúde.

A partir da realização deste estudo, verificou-se que pesquisas devem ser promovidas com o intuito de subsidiar a construção de conhecimentos sólidos no que se refere à presença e a atuação do profissional de Fonoaudiologia em Minas Gerais. Verifica-se a necessidade crescente de que sejam desenvolvidos estudos de base epidemiológica ${ }^{27}$, bem como pesquisas que apontem as reais condições de saúde fonoaudiológicas da população, de modo a orientar a alocação de fonoaudiólogos nas regiões e a despertar a atenção de gestores para a importância deste profissional como componente das equipes de Atenção à Saúde em todas as áreas.

\section{CONCLUSÃO}

Observou-se aumento considerável na distribuição dos profissionais de Fonoaudiologia no estado de Minas Gerais no período de 2005 a 2010, passando de 1.237 em 2005 a 2.555 profissionais no ano de 2010.

Do total de municípios, somente 278 possuíam o profissional fonoaudiólogo na Atenção à Saúde em 2005. Cinco anos depois, observou-se o aumento de 186 municípios com fonoaudiólogo e 54,3\% dos municípios passaram a contar com o fonoaudiólogo na assistência.

A média de fonoaudiólogos por 10.000 habitantes passou de 0,61 em 2005 para 1,30 em 2010 no estado, indicando maior disponibilidade deste profissional para a população mineira.

\section{AGRADECIMENTOS}

Agradecemos à fonoaudióloga Fernanda Maciel pela colaboração no estudo. 


\section{ABSTRACT}

Purpose: to analyze trends in the distribution of speech therapists in Minas Gerais from 2005 to 2010. Method: it is a retrospective study, in which a secondary database of public domain - Reports in Health Information of Brazilian Health Information System, called DATASUS was analysed. It was considered data from 853 municipalities in Minas Gerais, highlighting the resource indicators related to the number of speech therapist, the number of professionals in the Minas Gerais macro regions, the population of these spaces, the binding of the speech therapists to the Public Health System (SUS) and the trends distribution of these professionals from 2005 to 2010. Results: from 2005 to 2010, there was a significant increasing in the number of speech therapists. The number of municipalities with speech therapist raised from $32,8 \%$ to $54,5 \%$. Since 2008 , marked as the peak of professional growth, there was an increase about $25 \%$. The number of speech therapist per 10.000 inhabitants increased from 0,61 in 2005 to 1,30 in 2010. Conclusion: there was a considerable increase in the distribution of speech therapists in the searched state, rising from 1237 professionals in 2005 to 2555 professionals in 2010. In 2005, 278 of the municipalities had a speech therapist in the health care. Five years later, there was an increase of 186 municipalities with speech therapist, indicating greater availability of this professional for the mining population. Although the data from this study reinforce the accessibility and professional presence in Minas Gerais, it was notable regional discrepancy in the state.

KEYWORDS: Speech, Language and Hearing Sciences; Unified Health System; Family Health Program; Public Health; Public Policies

\section{REFERÊNCIAS}

1. Silva MEML, Brasil CCP, Regis ACF. Desafio do Núcleo de Atenção Médica Integrada diante da Necessidade de inserção de fonoaudiólogo na rede municipal de Saúde de Fortaleza. Saúde soc. 2010;19(4):838-51.

2. Penteado RZ, Servilha EAM. Fonoaudiologia em saúde pública/coletiva: compreendendo prevenção e o paradigma da promoção da saúde. Distúrbios da Comunicação. 2004;16(1):107-16.

3. Bazzo LMF. Privação da oferta de serviços fonoaudiológicos no Sistema Único de Saúde (SUS) e a reforma do Estado: a mediação do Debate. R. C. méd. biol., Salvador. 2007;6(2):190-6.

4. BRASIL. PORTARIA GM/MS № 2.073, de 28 de setembro de 2004. Institui a Política Nacional de Atenção à Saúde Auditiva. http://bvsms.saude. gov.br/bvs/publicacoes/politica_nacional_atencao_ saude_auditiva.pdf

5. BRASIL. Portaria GM/MS № 154, de 24 de janeiro de 2008. Cria os Núcleos de Apoio à Saúde da Família. Disponível em: http://200.137.177.147/ sistemas_de_informacao/doc_tec_leg/siab/ portaria-n-154-nasf.pdf

6. Moreira MD, Mota HB. Os Caminhos da Fonoaudiologia no Sistema Único de Saúde-SUS. Rev. Cefac. 2009;11(3):516-21.

7. Cavalheiro MTP. Fonoaudiologia e saúde da família. Rev. CEFAC. 2009;11 (2):4-5.
8. Freire RM. Fonoaudiologia em saúde pública. Rev. Saúde Pública. 1992;26 (3):179-84.

9. Lipay MS, Almeida EC. A fonoaudiologia e sua inserção na saúde pública. Rev. Ciênc. Méd. 2007;16(1):31-41.

10. Pereira FCB, Aarão PCL, Seixas KL, Silva HG, Tavares APN, Campos FR et al. Histórico da Fonoaudiologia em Minas Gerais: Impressão dos Protagonistas. Rev. CEFAC. No prelo. 2011.

11. Narvai PC, Frazao P, Roncalli AG, Antunes JLF. Cárie dentária no Brasil: declínio, polarização, Iniqüidade e Exclusão sociais. Rev Panam Salud Publica. 2006;19(6):385-93.

12. Bevilacqua MC, Morettin M, Melo T, Amantini RCB, MartinezMaria ANS. Contribuições para análise da política de saúde auditiva no Brasil. Rev. soc. bras. fonoaudiol. 2011;16(3):252-9.

13. Molini-Avejonas DR, Mendes VLF, Amato CAH. Fonoaudiologia e Núcleos de Apoio à Saúde da Família: conceitos e referências. Rev. Soc Bras Fonoaudiol. 2010;15(3):465-74.

14. Johnson A. Promoting the Value of Audiology and Speech-Language Pathology in Health Care. The ASHA Leader. 2006.

15. Santos JN, Maciel FJ, Martins VO, Rodrigues ALV, Gonzaga AF, Silva LF. Inserção dos fonoaudiólogos no SUS/MG e sua distribuição no território do estado de Minas Gerais. Rev. CEFAC. No prelo 2011. 
16. Lessa FJD, Miranda GMD. Fonoaudiologia e Saúde Pública. In: Britto ATB de (Org.). Livro de Fonoaudiologia. São Jose dos Campos: Pulso Editorial, 2005.

17. BRASIL- Lei $n^{\circ} 2.192 / 03$, de 15 de maio de 2010. Dispõe sobre a jornada de trabalho do Fonoaudiólogo.

18. Ministério da Saúde. DATA SUS. Caderno de Informações em Saúde do estado de Minas Gerais, versão fevereiro de 2009. Disponível em: www. datasus.gov.br.

19. Somefun OA, Lesi FEA, Danfulani MA, Olusanya BO. Communication disorders in Nigerian children. International Journal of Pediatric Otorhinolaryngology. 2006;70:697-702.

20. Fernandes EL, Cintra LG. A Inserção da Fonoaudiologia na Estratégia da Saúde da Família: relato de caso. Rev. APS. 2010;13(3):380-5.

21. Furtado LM, Almeida EC. Acolhimento em Saúde Pública: A Contribuição do Fonoaudiólogo. Rev. Ciênc. Méd. 2006;15(3):249-56.

22. Casanova IA, Moraes ALA, Ruiz-Moreno L. $O$ ensino da promoção da saúde na graduação de fonoaudiologia na cidade de São Paulo. Pro-Posições 2010;21(3):219-34.

23. Lemos M, Bazzo LMF. Formação do fonoaudiólogo no município de Salvador e consolidação do SUS. Ciênc. saúde coletiva. 2010;15(5):2563-8.

24. Batella WB, Diniz AMA. Desenvolvimento humano e hierarquia urbana: uma análise do IDH-M entre as cidades mineiras. Revista de Biologia e Ciências da Terra. 2008; 6(2):367-74.

25. Verdon S, Wilson L, Smith-Tamaray $M$, McAllister L. An investigation of equity of rural speech-language pathology services for children: a geographic perspective. Int J Speech Lang Pathol. 2011;13(3):239-50.

26. Lima BPS, Guimarães JATL, Rocha MCG. Características epidemiológicas das alterações de linguagem em um centro fonoaudiológico do primeiro setor. Rev. Soc. Bras. Fonoaudiol. 2008;13(4):376-80.

27. Pieter GN. The ECHI Project: Health indicators for the European Community. European Journal of Public Health. 2003;13(3):101- 6.

http://dx.doi.org/10.1590/S1516-18462013005000011

Recebido em: 18/01/2012

Aceito em: 16/04/2012

Endereço para correspondência:

Juliana Nunes Santos

Rua Coronel Pedro Jorge, 170/201 - Prado

Belo Horizonte - MG - Brasil

CEP: 30410-350

E-mail: jununessantos@yahoo.com.br 